\title{
BALANCING STOCHASTIC KNOWLEDGE ON ACOUSTICS AND LINGUISTICS
}

\author{
Dept. of Information Electronics \\ Nagoya University \\ NAGOYA, 465 JAPAN
}

Kazuya TAKEDA, Atsunori OGAWA and Fumitada ITAKURA

\begin{abstract}
Since basic processing units, i.e. the speech segment and the word, are different, careful balancing between acoustic and linguistic knowledge is needed in stochastic speech recognition systems. In most systems, combining acoustic and linguistic scores is controlled by a heuristic parameter, language weight. In order to achieve a robust balance, taking sequence length into account is important because the length of a speech segment is much shorter than a word duration. The length of the word sequence is not taken into account under n-gram language modeling, which is the most common stochastic language modeling for speech recognition. Due to this property, the optimal values of the language weight and word insertion penalty for balancing acoustic and linguistic probabilities is affected by the length of word sequence. To deal with this problem, a new language model is developed based on Bernoulli trial model taking the length of the word sequence into account. Not only better recognition accuracy but also robust balancing with acoustic probability compared with the normal n-gram model of the proposed method is confirmed through recognition experiments.
\end{abstract}

\section{INTRODUCTION}

The basic scheme of stochastic speech recognition is to find a word sequence which maximizes the conditional probability given by

$$
P(W \mid A)=\frac{P(A \mid W) P(W)}{P(A)},
$$

across the possible word sequence $W$ given acoustic observation $A$. A merit of stochastic approaches for continuous speech recognition is not only that it can be trained by using a large amount of training data, but also the ease of combining two or more different knowledge sources [5]. In fact, disregarding the denominator term, as it does not contribute the relative probability, the above basic formula turns to be a simple form of multiplying two probabilities given by acoustic knowledge (probability density function) $P(A \mid W)$ and linguistic knowledge (a priori probability) $P(W)$, i.e.

$$
\log P(A, W)=\log P(A \mid W)+\log P(W),
$$

for combining two different knowledge sources. However, in practice, this simple multiplication needs to be modified for balancing the absolute values of two probabilities [1],[9],[10].

The most common modification for balancing two probabilities is to use a language weight $\alpha$ and insertion penalty $Q$, i.e.

$$
\log \hat{P}(A, W)=\log P(A \mid W)+\alpha\{\log P(W)-n Q\},
$$

where $n$ is the word length of the sequence $W$. (An alternative formulation

$$
\log P(A \mid W)+\alpha \log P(W)-n Q
$$

is adopted in some systems such as HTK[11]. In this case, the dependency between $Q$ and $\alpha$ discussed below is greater.) Since the probabilistic meaning of these two parameters is not clear, both of them are determined experimentally.

The purpose of this paper is to discuss and solve the three problems of this heuristic balancing; 1) the balancing parameters are critical to recognition accuracy; 2) the optimal values of two parameters are related with each other; and 3) the optimal values are also governed by the length of word sequence. In the rest of this paper, we first present the above mentioned problems from experimental results. Then, we propose n-gram-Bernoulli language modeling, which is a simple extension of n-gram model but takes the length of the word sequence into account, for removing the sequence length dependency from the combining process. Finally, we will show the effectiveness of the proposed modeling by experimental results. 


\section{PROBLEMS IN COMBINING PROBABILITIES}

In n-gram language modeling, the probability of an nword-long partial word sequence is given as the $\mathrm{n}$-time product of the local probabilities. The simple consequence of this modeling is that a lower probability is assigned to a longer word sequence, i.e.

$$
P\left(\mathbf{w}_{1}^{n}\right) \geq P\left(\mathbf{w}_{1}^{n+1}\right) .
$$

For combining with acoustic probability, a problem arises due to this simple decreasing property of n-gram. When the language probability is weighted too heavily, due to preference of shorter word sequences in n-gram modeling, the output of the recognizer is likely to be shorter than the correct answer. Thus the optimal value of $\alpha$ depends on the word-length of the sentence to be recognized. Experimental evidence of this shortening is shown in Figure 1 (a) as the averaged length of recognition results versus $\alpha$.

Word Insertion Penalty (WIP) is used as another heuristic in order to compensate for the word length dependency of $n$-gram modeling, as follows. When the language probability is not weighted enough, or $\alpha$ is optimized for shorter sentences, giving a penalty proportional to the word length helps to inhibit word insertions. Instead, when language probability is weighted too heavily, negative WIP works to inhibit word deletion errors. Thus we have to optimize $\alpha$ for the difference in scale of acoustic and linguistic probabilities and then optimize $Q$ for the particular $\alpha$. This is also confirmed experimentally as shown in Figure 1 (b).

\section{LANGUAGE MODELING TAKING SENTENCE LENGTH INTO ACCOUNT}

\subsection{Unigram-Bernoulli modeling}

In order to incorporate the length of the word sequence in modeling, we introduce generalized Bernoulli trials as a basic model, which is defined as below. Suppose a set of $r$ disjoint events, $\mathcal{B}=\left\{\mathcal{B}_{1}, \mathcal{B}_{2}, \cdots, \mathcal{B}_{r}\right\}$, after $n$ independent trials, the probability of the observation that $\mathcal{B}_{i}$ occurs $k_{i}$ times, for $i=1 \cdots r$ in any order is calculated by

$$
P\left(k_{1}, k_{2}, \cdots, k_{r}\right)=\frac{n !}{\prod_{j=1}^{r} k_{j} !} \prod_{j=1}^{r}\left\{P\left(\mathcal{B}_{j}\right)\right\}^{k_{j}} .
$$

where $P\left(\mathcal{B}_{j}\right)$ stands for the probability of event $\mathcal{B}_{j}$ and $\sum_{i=1}^{r} k_{i}=n$ holds.

The first term $n ! / \prod_{j=1}^{r} k_{j}$ ! accounts for the number of combinations of event sequences constrained by the

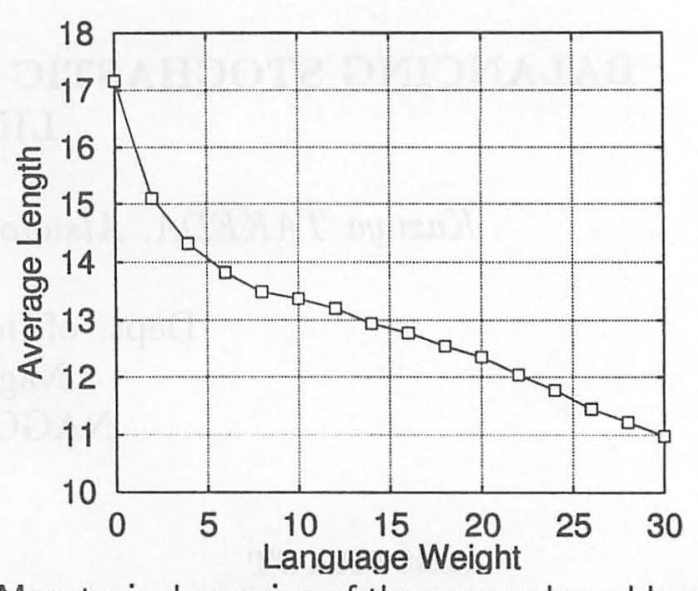

(a) Monotonic decreasing of the averaged word length of recognition results.

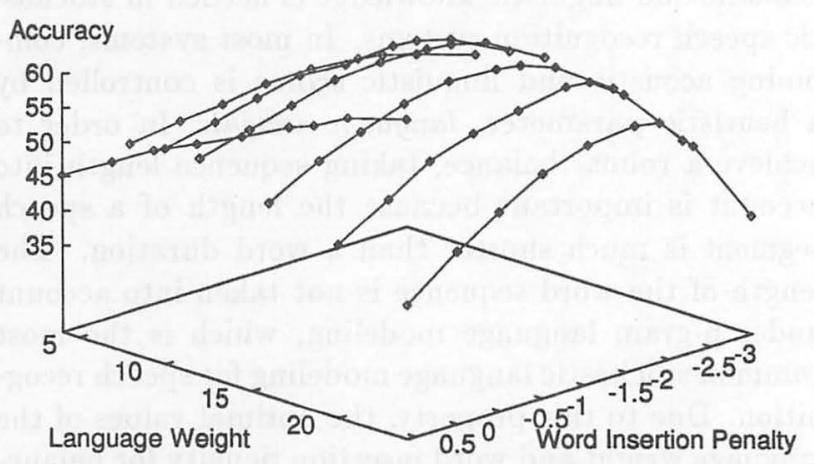

(b) Dependency between $\alpha$ and $Q$ in optimizing.

Figure 1: Problems of heuristic parameters in combing acoustic and linguistic probabilities. Recognition experiment results using bigram LM.

frequency of each event. Note that if each event occurs only once, the term becomes $n$ !, which represents the total number of possible orders of event sequences. The second term $\prod_{j=1}^{r}\left\{P\left(\mathcal{B}_{j}\right)\right\}^{k_{j}}$ calculates the probability of each ordered event sequence.

One can use the eqn. (2) as a stochastic language model by regarding the word occurrence as $\mathcal{B}_{i}$. Using the length of the word sequence as $n$, the Bernoulli trial probability

$$
P_{\text {UGBeR }}(\mathbf{W})=\frac{n !}{\prod_{j=1}^{L} k_{j}(\mathbf{W}) !} \prod_{j=1}^{L}\left\{P\left(w_{j}\right)\right\}^{k_{j}(\mathbf{W})}
$$

where $k_{j}(\mathbf{W})$ is the frequency of word $w_{j}$ in the word sequence $\mathbf{W}$, can be utilized as a language model taking the sequence length into account. Since $P\left(w_{j}\right)$ is the unigram probability, we call the model the UnigramBernoulli language model. 


\subsection{Extending to n-gram}

Unigram-Bernoulli model takes sentence length into account, however, unigram does not provide any constraint concerning word order. Thus, we try to replace unigram with n-gram local probabilities. In n-gram cases, the event space becomes a Cartesian product of $\mathcal{B}$. In the bigram case, for example, each trial is associated with word occurrence conditioned by the previous word. This can be realized by assigning $P\left(w_{i} \mid w_{i-1}\right)$ for $P\left(\mathcal{B}_{i}\right)$, word length $n$ for number of trials and occurrence of each word pair in the sequence as $k_{j}$, i.e.

$$
P_{\text {BGBER }}(\mathbf{W})=\frac{n !}{\prod_{l=1}^{L} \prod_{m=1}^{L} k_{l \mid m}(\mathbf{W}) !} \prod_{i=1}^{n} P\left(w_{i} \mid w_{i-1}\right) .
$$

From the above mathematical formula, it is clear that the n-gram-Bernoulli model is a sequence length dependently weighted version of the conventional ngram language models. Thus, although the formulation started with the assumption of independent trials, n-gram-Bernoulli modeling is expected to provide local word-order constraints as well as compensation of the monotonic decreasing against the word length in simple n-gram language model.

\subsection{Implementation}

In practice, the frequency of a particular word-pair in a sentence is almost always either 0 or 1 (and $0 !=$ 1 ! = 1), neglecting the denominator leads the recursive calculation form for the n-gram-Bernoulli model, i.e.

$$
\begin{aligned}
\log \hat{P}_{\mathrm{BGBER}}\left(\mathbf{W}_{1}^{m}\right) & =\log n+\log P\left(w_{n} \mid w_{n-1}\right) \\
& +\log \hat{P}_{\mathrm{BGBER}}\left(\mathbf{W}_{1}^{n-1}\right)
\end{aligned}
$$

From the above form, it can be seen that the additional factor in calculating n-gram-Bernoulli probability is only the logarithm of the total word length from the beginning of the sequence.

\subsection{Relation with Insertion Penalty}

As shown in Figure 1, negative WIP improves recognition accuracy in most cases and it can be related with (the enumerator $n$ ! of the weighing term in) n-gramBernoulli modeling in the following way,

$$
n Q=n \log q \leftrightarrow n \log n \approx \log (n !)
$$

Therefore, one can see that negative WIP is included in the n-gram-Bernoulli modeling, and no explicit WIP, needs to be incorporated in n-gram-Bernoulli modeling.
Table 1: Analysis conditions

\begin{tabular}{|l|l|}
\hline Sampling frequency & $16 \mathrm{kHz}$ \\
\hline Quantization bit & $16 \mathrm{bit}$ \\
\hline Window type & Hamming \\
\hline Frame length & $25 \mathrm{msec}$ \\
\hline Frame period & $10 \mathrm{msec}$ \\
\hline Pre-emphasis & 0.97 \\
\hline MFCC order & 12 \\
\hline$\Delta$ MFCC order & 12 \\
\hline$\Delta$ power order & 1 \\
\hline
\end{tabular}

\section{EXPERIMENTAL EVALUATIONS}

\subsection{Experimental Setup}

The effectiveness of n-gram-Bernoulli modeling is confirmed by comparing with a simple n-gram model and a normalized version of the n-gram model. The normalized version of the n-gram probability is given by

$$
P_{\text {NGNOR }}(\mathbf{W})=\left\{\prod_{i=1}^{n} P\left(w_{i} \mid \mathbf{w}_{i-N+1}^{i-1}\right)\right\}^{1 / n},
$$

where $n$ is the length of word sequence.

n-gram probabilities are trained by ATR travel conversation corpora, containing 7,740 sentences with a vocabulary size of 4,784 words. All text is tagged using 27 POS categories. As the size of the corpora is not large, we used POS category based n-gram for calculating the word-n-gram. For example a word bigram is given by

$$
P\left(w_{i} \mid w_{i-1}\right) \approx P\left(c_{i} \mid c_{i-1}\right) P\left(w_{i} \mid c_{i}\right)
$$

where $w_{i}$ falls into a POS category $c_{i}$.

Triphone HMM's were used as the acoustic model. The HMM has 3 states and each state has $4 \mathrm{mix}$ tures. Total number of states is approximately 12,000 . We trained this model using 8,128 utterances from 54 speakers in the continuous speech corpus for research of the Acoustical society of Japan. Configurations of acoustic analysis conditions are listed in Table 1.

For the test utterance, one male speaker uttered a total of 150 sentences extracted from the training corpus. The test set consists of 5 subsets. Each subset contains 30 sentences of the same word-length, i.e. 9, $12,15,18,21$ words-long.

\subsection{Results}




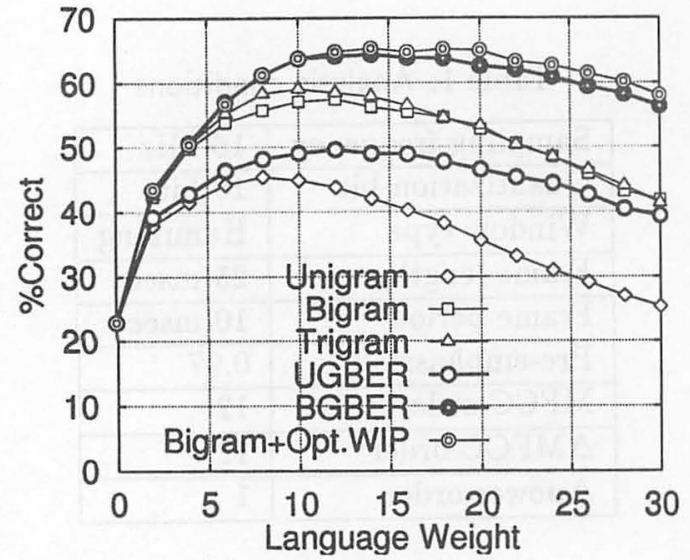

(a) Word Correct rate

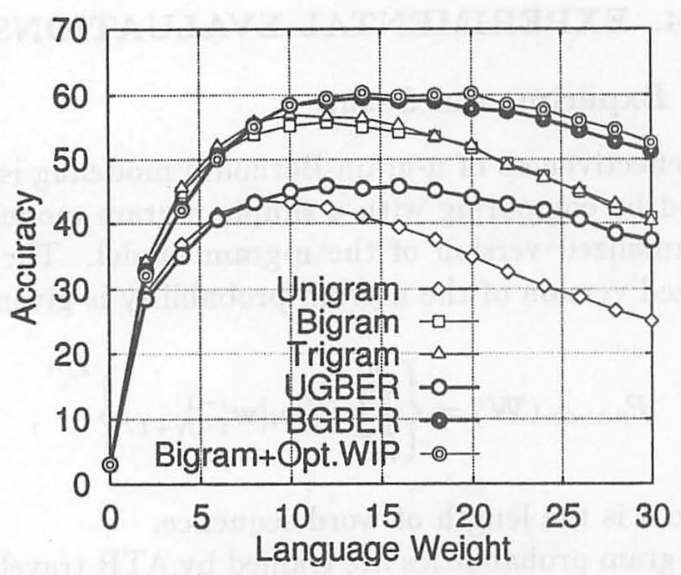

(b) Word Accuracy Score

Figure 2: Recognition performance across the language weight values. ( $\mathrm{N}$ gram and $\mathrm{N}$ gram-Bernoulli models.)

Results of the experiments are given in Figure 2, 3 and 4. Throughout the figures, each model is referred as UGBER: Unigram-Bernoulli, BGBER: BigramBernoulli, UGNOR: normalized version of Unigram, BGNOR: normalized version of Bigram.

Figure 2 shows that the performance of the n-gramBernoulli model is better than the simple n-gram at all values of language weight. The performance of the Bigram-Bernoulli model is better than simple trigram results. Furthermore, the range of the language weight which gives the best recognition performance is wider in n-gram-Bernoulli than the simple n-gram model. With comparing to the case using WIP, the results of n-gramBernoulli model is comparable to the case when both language weight and WIP are carefully optimized by experiment. Furthermore, the range of the language weight which gives the best recognition performance is wider in n-gram-Bernoulli than the simple n-gram
Table 2: Weight range for giving $\{$ maximum -5$\}[\%]$

\begin{tabular}{|c|c|c|}
\hline & \%Corr (UG,BG) & Acc (UG,BG) \\
\hline n-gram & $4.0 \sim 14.0,6.0 \sim 20.0$ & $6.0 \sim 16.0,6.0 \sim 20.0$ \\
\hline BER & $6.0 \sim 22.0,8.0 \sim 26.0$ & $8.0 \sim 24.0,8.0 \sim 24.0$ \\
\hline
\end{tabular}

model, as listed in Table 2.

The recognition performance of the normalized-ngram probability is shown in Figure 3. Note that the language weight value is different from the previous experiments. It can be seen that the word correct rate of the normalized n-gram is comparable with the bigramBernoulli model. However, the word accuracy score is much lower than the n-gram-Bernoulli model. This result clarifies that the per-word evaluation of language probability causes frequent insertion errors.

The characteristics of each model can be discussed from the viewpoint of the average word length of recognition output, which is shown in Figure 3. The monotonic decreasing in average word length of the simple n-gram model is not significant in both the n-gramBernoulli and the normalized n-gram. In the normalized n-gram, however, the averaged word length is longer than the real length (15 words in this experiment) due to frequent word insertion errors.

\section{SUMMARY}

In this paper we have discussed balancing acoustic and linguistic probabilities by extending the n-gram language modeling by taking the length of the word sequence into account. The basic framework of the modeling is to extend Bernoulli trial modeling of word occurrence to that of being conditioned by previous words. The effectiveness of the modeling is evaluated from the viewpoints concerned with the problems of simple n-grams, 1) the balancing parameters are critical to recognition accuracy; 2) the optimal values of two parameters are related with each other; and 3) the optimal values are also governed by the length of word sequence. The experimental results show that the ngram-Bernoulli modeling is effective for dealing with those problems.

\section{REFERENCES}

[1] L. R. Bahl, R. Bakis, F. Jelinek and R. L. Mercer, "Language-model/acoustic channel balance 


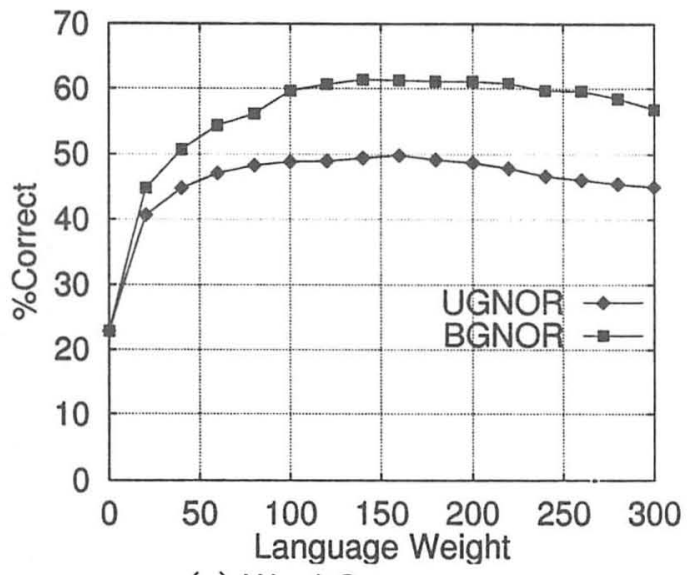

(a) Word Correct rate

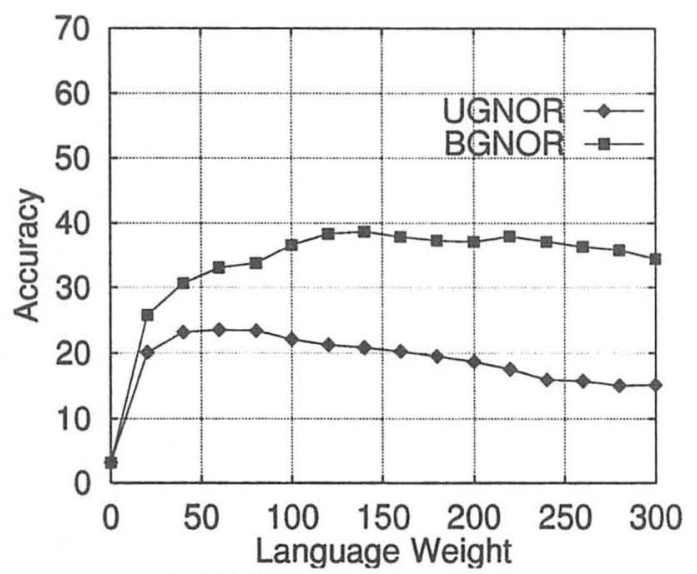

(b) Word Accuracy Score

Figure 3: Recognition performance across the language weight values. (Normalized $\mathrm{N}$ gram models.)

mechanism", IBM Technical Disclosure Bulletin, 23(7B), pp.3464-3465, Dec. 1980.

[2] J.Bellegarda, J.Butzberger, Y.Chow, N.Coccaro and D.Naik, "A Novel Word Clustering Algorithm Based on Latent Semantic Analysis," Proc. of ICASSP96, vol. 1, pp. I-172-175, May 1996.

[3] P.R.Clarkson and A.J.Robinson, "Language Model Adaptation using Mixtures and Exponentially Decaying Cache," Proc. of ICASSP97, vol. 2, pp. 799-802, April 1997.

[4] T. Ehara, N. Inoue, H. Kohyama, T. Hasegawa, F. Shohyama and T. Morimoto, "Contents of the ATR Dialogue Database", ATR Technical Report, Oct. 1990, in Japanese.

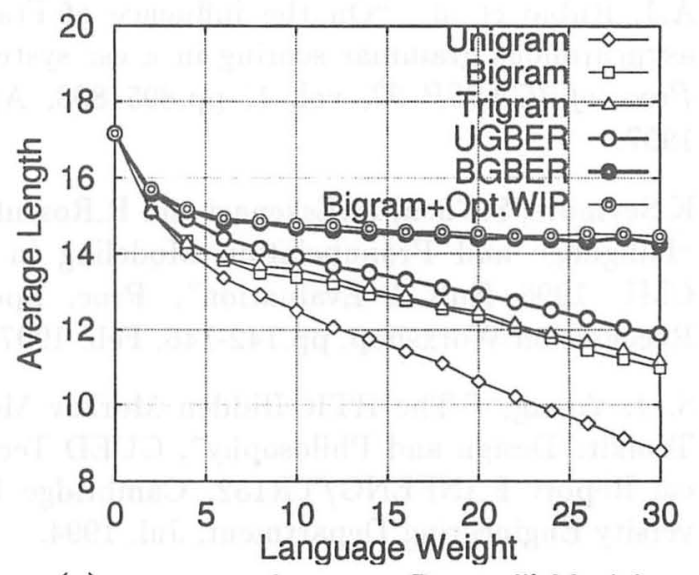

(a) n-gram and n-gram-Bernoulli Models

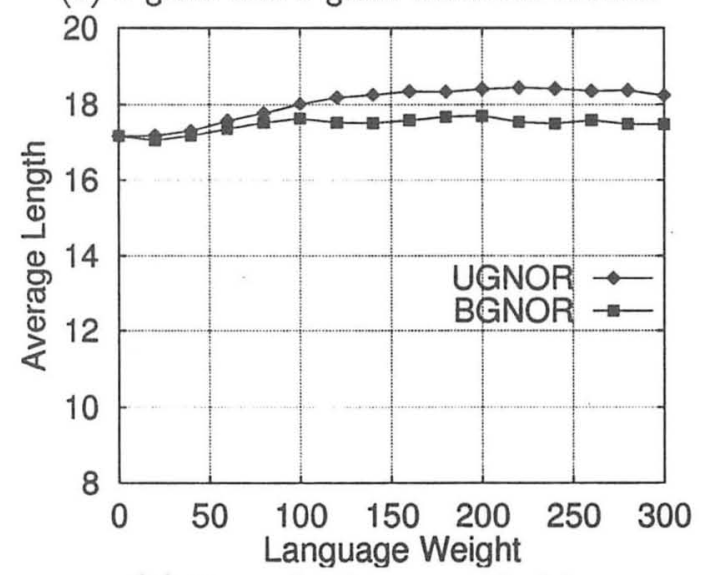

(b) Normalized n-gram Models

Figure 4: Average length of recognition result across the language weight values.

[5] F. Jelinek, "Self-organized language modeling for speech recognition", in Readings in Speech Recognition, Morgan Kaufmann Publishers, Inc. 1990.

[6] S. M. Katz, "Estimation of probabilities from sparse data for the language model component of a speech recognizer,", IEEE Trans. on Acoustic Speech and Signal Processing, vol. 35, no. 3, pp. 400-401, Mar. 1987.

[7] T. Kobayashi, S. Itabashi, S. Hayamizu and T. Takezawa, "ASJ continuous speech corpus for research", J. Acoust. Soc. Japan, Vol.48, No.12, pp.888-893, 1992, in Japanese.

[8] H.Masataki, Y.Sagisaka, K.Hisaki and T. Kawahara, "Task adaptation using MAP estimation in $\mathrm{N}$-gram language modeling," ICASSP97, vol. 2, pp. 783-786, April 1997. 
[9] A.J. Rubio et al., "On the influence of Frameasynchronous grammar scoring in a csr system," Proc. of ICASSP 97, vol. 1, pp.895-898, April. 1997.

[10] K.Seymore, S.Chen, M.Eskenazi and R.Rosenfeld, "Language and Pronunciation Modeling in the CMU 1996 Hub 4 Evaluation", Proc. Speech Recognition Workshop, pp.142-146, Feb. 1997.

[11] S. J. Young, "The HTK Hidden Morkov Model Toolkit: Design and Philosophy", CUED Technical Report FINFENG/TR152, Cambridge University Engineering Department, Jul. 1994. 